\title{
Measuring sedentary behaviours in patients with idiopathic pulmonary fibrosis using wrist-worn accelerometers
}

\begin{abstract}
Introduction - Idiopathic pulmonary fibrosis (IPF) patients suffer increasing functional limitation with disease worsening disease. Increasing time in sedentary behaviour has been associated with poorer quality of life. Determining thresholds for activity in patients with respiratory disease is difficult due to variable cardiorespiratory limitations between individuals. Measuring sedentary behaviour is not confounded by this limitation and may be a better measurement of activity in patients with respiratory disease.
\end{abstract}

Objectives - To measure sedentary time in patients with IPF using wrist-worn accelerometers.

Methods - 39 IPF patients wore a GENEActiv actiwatch continually for 7 days. Participants underwent measurement of forced vital capacity, diffusion capacity of carbon monoxide and 6 minute walk distance.

Results - Valid data was captured from 35 of 39 participants (89.7\%). Mean acceleration intensity recorded in the most active 5 hours of each day (in milli-g) were 43.8 milli-g and sedentary time was 551.7 minutes per day. Daily sedentary time correlated moderately with M5 values (Pearson correlation $-0.366, p=0.030)$. Only M5 values predicted sedentary time. No variability in sedentary time was seen by day of the week. There was a trend towards higher one and two-year mortality with increasing sedentary time.

Conclusions - Wrist-worn accelerometers reliably collected data and were well tolerated. IPF patients spent long periods of time in sedentary behaviours. Of the standard clinical measures used, 6MWD predicted daily activity but not sedentary time; no clinical measures predicted sedentary time. Increased sedentary time may be associated with poorer outcomes in IPF patients.

Keywords: Exercise, Idiopathic Pulmonary Fibrosis, Pulmonary Fibrosis, Interstitial Lung Disease 


\section{Measuring sedentary behaviours in patients with idiopathic pulmonary fibrosis using wrist-worn accelerometers}

\section{Introduction}

Idiopathic pulmonary fibrosis (IPF) is a progressive fibrosing interstitial lung disease (ILD) of unknown cause $^{1}$. Affected lungs show characteristic fibrosis, with progressive impairment of gas transfer leading to exercise-induced dyspnoea ${ }^{2}$ and reduced physical activity in daily life ${ }^{3}$. Physical activity is associated with numerous health benefits ${ }^{4}$. However, it is becoming increasingly apparent that prolonged time in sedentary behaviours, inactivity defined as a combination of sitting and low levels of energy expenditure ${ }^{5}$, has negative effects on health even if recommendations on physical activity levels are met $^{6-8}$. This may be of particular importance in patients with chronic lung diseases, including IPF, where achieving recommended levels of moderate or vigorous physical activity may be unrealistic due to exertional dyspnoea and fatigue ${ }^{9}$.

Daily activity in free-living conditions can be measured over a period of several days using multi-axis accelerometers. The accuracy of these devices to estimate energy expenditure compared with indirect calorimetry or doubly labelled water has been investigated in certain chronic lung diseases ${ }^{10}$ but not in patients with interstitial diseases like IPF. However, wrist-worn accelerometers are also able to measure sedentary behaviours using postural data ${ }^{11}$. In contrast to the difficulties of classifying activity vigour using accelerometer outputs in disease, we hypothesised that the accuracy of measuring sedentary behaviour time with these devices should not significantly vary between health and disease. Our rationale for this was that the definition of sedentary behaviours will not alter in patients with chronic disease as the two defining aspects of sedentary behaviours (sitting position and low energy state) should remain unchanged even with marked cardiorespiratory limitation. 
This study investigates the feasibility of using a wrist-worn, three-axis accelerometer as a measure of daily physical activity and time in sedentary behaviours in patients with IPF, and evaluates the associations between time spent in sedentary behaviours and lung function, six minute walk distance (6MWD) and questionnaire measures of dyspnoea, depression and anxiety, fatigue and quality of life, as well as accelerometer outputs for activity levels.

\section{Methods}

\section{Subjects}

Patients with a diagnosis of definite or probable IPF based on high resolution CT criteria $^{1}$, or lung biopsy confirming usual interstitial pneumonia (UIP) without an alternative cause, were approached in the respiratory clinic at Norfolk and Norwich University Hospital, Norfolk UK. Ethical approval was given by South Central - Oxford C research ethics committee (reference number 13/SC/0116). Patients were excluded if they had significant cardiac or pulmonary disease contributing to their symptoms, respiratory tract infection within 4 weeks of inclusion, significant co-morbid disease that was likely to impact on daily activity, or inability to consent.

Forty-eight patients consented to participate. Four patients were subsequently found to have diagnoses other than IPF and five patients withdrew consent before undertaking any assessments. In total, thirty-nine participants underwent clinical assessment and daily activity measurement. All included patients were clinically stable during the measurement period.

\section{Clinical Assessments}

Prior to activity measurement, all participants underwent 6MWD, and pulmonary function tests (PFTs). PFTs were performed in the pulmonary function laboratory at the Norfolk and Norwich Hospital (CareFusion Masterscreen PFT system). The results of the forced vital capacity (FVC) and 
diffusing capacity of carbon monoxide (DLCO) were recorded as a percentage of predicted values. Patients were stratified by disease severity, with severe disease defined as an FVC $<55 \%$ or DLCO $<40 \%{ }^{12}$. The $6 \mathrm{MWD}$ was performed without supplemental oxygen and in accordance with ATS guidelines ${ }^{13}$. Baseline and minimum saturations during the $6 M W D$ were recorded. Participants completed a number of questionnaires: the MRC dyspnoea scale ${ }^{14}$ was performed to determine their severity of dyspnoea, the Hospital Anxiety and Depression Scale (HADS), which is able to identify and quantify two common forms of psychological problems in patients with ILD ${ }^{15}$, the Fatigue Assessment Scale (FAS) ${ }^{16}$ and the Kings Brief Interstitial Lung Disease (KBILD) questionnaire ${ }^{17}$. Mortality data for participants was recorded from their hospital records for 24 months following participation.

Measurement of daily activity

All participants were asked to wear a wrist-worn, tri-axial accelerometer (GENEActiv actiwatch; GENEActiv, Cambridgeshire, UK) on their non-dominant wrist continuously for at least 7 consecutive days. The sampling frequency of the device was $50 \mathrm{~Hz}$. Data from the devices was defined as valid if there was at least 16 hours of data on at least 2 week days and 2 weekend days ${ }^{18}$. Accelerometer data was processed according to acceleration intensity and time in sedentary behaviour. Activity data was analysed using the $R$-statistics package $\mathrm{GGIR}^{19}$. Mean acceleration intensity (the single vector magnitude of the combined accelerations in all three axes recorded by the device) in the most active 5 hour period in each day was recorded in milli-g units (M5) and averaged across each day of valid wear time. Time in sedentary behaviour was calculated by converting raw data from the GENEActiv devices to 15 second epoch files by the GENEActiv PC software and then analysed using a sedentary sphere custom spreadsheet (available from the authors of the original paper ${ }^{11}$ ). 
Data analysis was undertaken using SPSS Statistics version 22 (IBM Corp, Illinois, USA). Data was divided into terciles by average minutes in sedentary behaviours per day. Comparison of M5 and mean daily time in sedentary behaviours were compared after stratifying IPF severity by lung function values, using FVC $<55 \%$ predicted or DLCO $<40 \%$ predicted as per previous trials ${ }^{12}$. Time in sedentary behaviour was analysed by day of the week to investigate differences between weekdays and weekend days.

Bivariate analyses between predictor variables (age, FVC \% predicted, DLCO \% predicted, 6MWD, MRC dyspnoea score, HADS score, KBILD and FAS) and both M5 values and time in sedentary behaviours were performed. The M5 variable was logarithmically-transformed to normalise distribution (other variables were normally distributed). Subsequently, variables with $p<0.2$ in the bivariate analysis were introduced into a multivariate linear regression model with stepwise removal of non-significant predictors. Partial $r^{2}$ values for significant predictors remaining within the final model were calculated to determine variability explained by each predictor.

Mortality data for the 12- and 24-month period following study participation was analysed using the Kaplan-Meier estimator, with survival results displayed for each tercile of time spent in sedentary behaviours.

\section{Results}

Of the 39 patients who wore an accelerometer, 35 devices recorded valid data (89.7\%). Four devices contained insufficient data to analyse; two devices failed (battery insufficiently charged) and two devices were removed prematurely by participants before sufficient data had been collected.

Demographic and activity data for the participants are shown in Table 1. No patients were receiving supplemental oxygen at the time of assessment. Eleven patients (31.4\%) had severe disease. Exercise tolerance (6MWD) was moderately reduced. HADS scores were higher in the highest two 
terciles of time in sedentary behaviours. No differences in FAS scores were seen across each tercile and KBILD scores decreased across terciles of increasing time in sedentary behaviour suggesting worse health-related quality of life. The most sedentary group spent 195.4 minutes more time in sedentary behaviours per day on average than the least sedentary group. A trend towards higher accelerometer outputs during the most active 5 hours each day was seen, with the most sedentary group showing mean acceleration value of 36.9milli-g compared with 56.0 milli-g in the least sedentary group. There was a moderate negative correlation between M5 values and time in sedentary behaviour per day in the bivariate correlation $(r=-0.366, p=0.030)$. No differences were seen in M5 values or time in sedentary behaviour between patients with and without severe disease (Figure 1). No difference in time spent in sedentary behaviour was seen between any days of the week (Figure 2).

The outcomes from the bivariate correlation and the multivariate regression model are shown in Table 2. None of the clinical measures (lung function, 6MWD or questionnaire results) showed a relationship with time in sedentary behaviour. Only M5 values from the accelerometer showed significant correlation with time in sedentary behaviour; in the regression analysis this was the only variable that satisfied the requirements for inclusion in the model. This variable was responsible for only $13.4 \%$ of the variability in time in sedentary behaviour seen $\left(r^{2}=0.134\right)$. When predicting physical activity during the most active 5 hours, the 6MWD and FVC were the only variables that remained in the regression model, with $23.5 \%$ of the variability in the M5 value was predicted by these two variables.

Mortality data for the two years following participation in the study is shown in Figure 3. Six (17.1\%) patients died within one year of completing the study. Nine patients $(25.7 \%)$ died within two years. There was a tendency towards higher mortality in the two most sedentary terciles, although there was no significant statistical difference between the three terciles. 


\section{Discussion}

To our knowledge this is the first study to investigate sedentary behaviours in IPF patients using a wrist-worn, three-axis accelerometry devices. The results suggest these devices can feasibly measure time in sedentary behaviour and activity over a period of at least seven days in patients with chronic respiratory disease. No difference was seen in duration of sedentary behaviour by day of the week, suggesting that it is not necessary to measure both weekend and week days in retired patients with IPF. Although we did not compare multiple devices here (e.g. step counters or hip-worn accelerometers), in previous studies wrist-worn devices have been shown to be acceptable to wearers ${ }^{20}$, leading to greater wear-time ${ }^{21}$. Concerns about lack of agreement between waist and wrist accelerometers have been raised, but used appropriately the classification of activity intensity has been shown to be in the same range as those reported for waist-worn devices ${ }^{22,23}$. Furthermore, only the wrist-worn GENEActiv device has been used so far to measure time in sedentary behaviours ${ }^{11,24}$.

There was a wide spread of time spent in sedentary behaviours in the group. There were insufficient participants in this trial to draw firm conclusions about whether sedentary behaviours influence mortality, but the trend towards increased mortality at both one and two years in the more sedentary participants raises the possibility of an association. Previous studies have shown that people with greater sedentary time have poorer health outcomes ${ }^{8}$. Furthermore, the cohort here appeared relatively sedentary compared to populations of equivalent age. One study measuring sedentary behaviours and monitoring mortality and morbidity in 93,676 women in the United States of America reported that $25.9 \%$ of participants aged $70-79$, similar to the age range included here, spent less than 4 hours per day in sedentary behaviours ${ }^{25}$. In addition, participants spending less than 4 hours per day in sedentary behaviours had better outcomes than those spending more than 11 hours in sedentary behaviour per day (similar to the mean time spent in sedentary behaviours in tercile 3 of our cohort, of 656.7 minutes per day). Reducing sedentary behaviours may be beneficial, 
but predicting those patients in clinic who are likely to be highly sedentary may be difficult.

Furthermore it will be difficult for patients with severe disease to increase even light activity and is unlikely to affect prognosis in these cases. No clinical measures within this group showed a relationship with time in sedentary behaviour, whereas variability in measures of activity (M5) was predicted well by 6MWD; direct assessment of sedentary behaviours in free living using monitoring devices may be required.

We deliberately avoided using pre-determined thresholds for activity vigour using the GENEActiv devices as these thresholds are likely to vary highly between individual patients depending upon disease severity. The measure of physical activity we chose for analysis was the M5 value; it was felt that this measure would best reflect the peak activity of the subject without being influenced by the relatively longer periods of sleep or sedentary behaviours. Without previous validation with indirect calorimetry or double-labelled water as a comparison in patients with ILDs, the classification of physical activity vigour using these devices may be inaccurate. By contrast, the definition of sedentary behaviour should not be significantly different between healthy individuals and patients such as those included in this study, and therefore should be measured equally accurately by the GENEActiv device as when used in healthy volunteers ${ }^{11,24}$. As a result, the measurement of sedentary time should not need revalidating in this population as the use of accelerometers to define exercise intensity would.

No studies have been performed to validate accelerometers as a measure of physical activity energy expenditure in IPF, although they have been performed in COPD. This showed that the SenseWear Pro armband could accurately measure energy expenditure in metabolic equivalents in laboratory conditions, albeit with a tendency to underestimate energy expenditure when walking and overestimate when performing daily activities ${ }^{10}$. Studies that have used accelerometers in IPF have used proximally worn devices ${ }^{3,26,27}$. Wallaert and colleagues used the SenseWear Pro armband and showed that there was a $45 \%$ decrease in exercise expenditure $>2.5$ metabolic equivalents per day 
compared with a sedentary healthy population, as well as an association between lower steps per day and increased mortality ${ }^{3}$. Bahmer and colleagues also used the SenseWear Pro armband to identify that fatigue, alongside 6MWD, were the strongest predictors of activity measured as steps per day ${ }^{27}$. In the paper by Wallaert and colleagues, 6MWD predicted $11 \%$ of the variability in number of steps per day, similar to the $13.7 \%$ in variability in M5 values explained by 6MWD in our study. Finally, Nakayama and colleagues used a waist-worn uniaxial accelerometer that had not previously been used in IPF patients, showing that only 70.6 minutes per day were spent performing any activity. This was the only study to describe potentially sedentary behaviours; 611.2 minutes per day were spent at a level of movement too low to measure as activity ( $<1$ magnitude of movement, corresponding with any activity less intense than light activity ${ }^{26}$. Because their device couldn't determine posture it is impossible to determine whether all of this time was truly sedentary. The time spent at this level of inactivity was slightly higher than observed in our participants (mean time in sedentary behaviour per day 551.7 minutes per day). The difference in time seen may relate to the different demographic studied, although the cohort in our study was older than the Japanese cohort (mean age 75.1 years compared with 68.3 years). Alternatively, inability to wear the waistworn device at all times may have caused under-recording of time spent performing sedentary behaviours but non-wear time was not reported in the paper.

The measurement of sedentary behaviour in patients with IPF and other chronic respiratory diseases may become increasingly important. Whilst the difficulty of increasing physical activity to the point of meeting recommended guidelines has been discussed in COPD patients ${ }^{9}$, the same problems affect patients with IPF. Chronic dyspnoea and fatigue limit exercise in this group ${ }^{27}$, and increasing peak exercise to spend more time performing at least moderate exercise may be unreasonable. There is some evidence to suggest that improving time in light activity compared with sedentary behaviour, even when not meeting recommended levels of moderate and vigorous intensity exercise, can improve various outcomes in healthy populations ${ }^{28}$; the benefits of increasing light 
physical activity and reducing sedentary behaviours in IPF and other chronic diseases warrants further investigation.

This study had a number of strengths. The IPF cohort analysed was well-characterised, excluded comorbidities that may have affected daily activity, and recording anxiety and depression scores. The number of participants providing valid data was high. The use of three-axis, wrist-worn accelerometer was able to provide large amounts of data, with participants wearing the devices for at least 7 days. The results reveal the sedentary behaviours of patients with IPF for the first time. There are also some weaknesses. The small number of participants within this feasibility study limits the ability to conclude whether increased time spent in sedentary behaviours is associated with worse outcomes. Further investigation with larger cohorts will be needed to investigate this association. There was a small range of HADS scores in our sample and therefore we may have underestimated the influence of this outcome on activity. Other factors that may help predict activity and sedentary behaviours, such as motivation and socio-economic factors, were not explored in this group.

In conclusion, the wrist-worn GENEActiv accelerometer can feasibly record sedentary behaviours in IPF patients. IPF patients spent significant periods of their day in sedentary behaviours. Of routine clinical measurements 6MWD was the best predictor of activity. A moderate inverse relationship between activity and sedentary behaviours was seen, but no clinical measurements could predict time in sedentary behaviour. Reducing sedentary time spent in and increasing time in light activities may be beneficial in IPF as it may improve outcomes and be a more achievable goal in patients who are markedly limited by their respiratory disease.

Acknowledgements: With thanks to Dr Alex Rowlands for his help regarding use of the sedentary sphere spreadsheet. 
Word Count: 2,742 


\section{References:}

1. Raghu G, Collard HR, Egan JJ, et al. An official ATS/ERS/JRS/ALAT statement: idiopathic pulmonary fibrosis: evidence-based guidelines for diagnosis and management. American journal of respiratory and critical care medicine. Mar 15 2011;183(6):788-824.

2. Meltzer EB, Noble PW. Idiopathic pulmonary fibrosis. Orphanet J Rare Dis. 2008;3:8.

3. Wallaert B, Monge E, Le Rouzic O, Wemeau-Stervinou L, Salleron J, Grosbois JM. Physical activity in daily life of patients with fibrotic idiopathic interstitial pneumonia. Chest. Nov 2013;144(5):1652-1658.

4. Kruk J. Physical activity in the prevention of the most frequent chronic diseases: an analysis of the recent evidence. Asian Pacific journal of cancer prevention : APJCP. Jul-Sep 2007;8(3):325-338.

5. Owen N, Healy GN, Matthews CE, Dunstan DW. Too much sitting: the population health science of sedentary behavior. Exercise and sport sciences reviews. Jul 2010;38(3):105-113.

6. Hamilton MT, Hamilton DG, Zderic TW. Role of low energy expenditure and sitting in obesity, metabolic syndrome, type 2 diabetes, and cardiovascular disease. Diabetes. Nov 2007;56(11):2655-2667.

7. de Rooij BH, van der Berg JD, van der Kallen CJ, et al. Physical Activity and Sedentary Behavior in Metabolically Healthy versus Unhealthy Obese and Non-Obese Individuals - The Maastricht Study. PloS one. 2016;11(5):e0154358.

8. Biswas $\mathrm{A}, \mathrm{Oh} \mathrm{PI}$, Faulkner $\mathrm{GE}$, et al. Sedentary time and its association with risk for disease incidence, mortality, and hospitalization in adults: a systematic review and meta-analysis. Annals of internal medicine. Jan 20 2015;162(2):123-132.

9. Hill K, Gardiner PA, Cavalheri V, Jenkins SC, Healy GN. Physical activity and sedentary behaviour: applying lessons to chronic obstructive pulmonary disease. Internal medicine journal. May 2015;45(5):474-482. 
10. Dhillon SS, Sima CA, Kirkham AR, Syed N, Camp PG. Physical Activity Measurement Accuracy in Individuals With Chronic Lung Disease: A Systematic Review With Meta-Analysis of Method Comparison Studies. Archives of physical medicine and rehabilitation. Nov 2015;96(11):2079-2088 e2010.

11. Rowlands AV, Olds TS, Hillsdon M, et al. Assessing sedentary behavior with the GENEActiv: introducing the sedentary sphere. Medicine and science in sports and exercise. Jun 2014;46(6):1235-1247.

12. Kolb M, Collard HR. Staging of idiopathic pulmonary fibrosis: past, present and future. European respiratory review : an official journal of the European Respiratory Society. Jun 2014;23(132):220-224.

13. Laboratories ATSCOPSfCPF. ATS statement: guidelines for the six-minute walk test. American journal of respiratory and critical care medicine. Jul 1 2002;166(1):111-117.

14. Mahler DA, Wells CK. Evaluation of clinical methods for rating dyspnea. Chest. Mar 1988;93(3):580-586.

15. Tzanakis N, Samiou M, Lambiri I, Antoniou K, Siafakas N, Bouros D. Evaluation of healthrelated quality-of-life and dyspnea scales in patients with idiopathic pulmonary fibrosis. Correlation with pulmonary function tests. Eur J Intern Med. Apr 2005;16(2):105-112.

16. De Vries J, Michielsen H, Van Heck GL, Drent M. Measuring fatigue in sarcoidosis: the Fatigue Assessment Scale (FAS). British journal of health psychology. Sep 2004;9(Pt 3):279-291.

17. Patel AS, Siegert RJ, Brignall K, et al. The development and validation of the King's Brief Interstitial Lung Disease (K-BILD) health status questionnaire. Thorax. Sep 2012;67(9):804810.

18. Sabia S, van Hees VT, Shipley MJ, et al. Association between questionnaire- and accelerometer-assessed physical activity: the role of sociodemographic factors. American journal of epidemiology. Mar 15 2014;179(6):781-790. 
19. van Hees VT, Gorzelniak L, Dean Leon EC, et al. Separating movement and gravity components in an acceleration signal and implications for the assessment of human daily physical activity. PloS one. 2013;8(4):e61691.

20. Huberty J, Ehlers DK, Kurka J, Ainsworth B, Buman M. Feasibility of three wearable sensors for 24 hour monitoring in middle-aged women. BMC women's health. 2015;15:55.

21. van Gestel AJ, Clarenbach CF, Stowhas AC, et al. Predicting daily physical activity in patients with chronic obstructive pulmonary disease. PloS one. 2012;7(11):e48081.

22. Welch WA, Bassett DR, Thompson DL, et al. Classification accuracy of the wrist-worn gravity estimator of normal everyday activity accelerometer. Medicine and science in sports and exercise. Oct 2013;45(10):2012-2019.

23. Zhang S, Rowlands AV, Murray P, Hurst TL. Physical activity classification using the GENEA wrist-worn accelerometer. Medicine and science in sports and exercise. Apr 2012;44(4):742748.

24. Rowlands AV, Yates T, Olds TS, Davies M, Khunti K, Edwardson CL. Sedentary Sphere: WristWorn Accelerometer-Brand Independent Posture Classification. Medicine and science in sports and exercise. Apr 2016;48(4):748-754.

25. Seguin R, Buchner DM, Liu J, et al. Sedentary behavior and mortality in older women: the Women's Health Initiative. American journal of preventive medicine. Feb 2014;46(2):122135.

26. Nakayama M, Bando M, Araki K, et al. Physical activity in patients with idiopathic pulmonary fibrosis. Respirology. May 2015;20(4):640-646.

27. Bahmer T, Kirsten AM, Waschki B, et al. Clinical Correlates of Reduced Physical Activity in Idiopathic Pulmonary Fibrosis. Respiration; international review of thoracic diseases. Jun 1 2016.

28. Loprinzi PD, Lee H, Cardinal BJ. Daily movement patterns and biological markers among adults in the United States. Preventive medicine. Mar 2014;60:128-130. 